\title{
Treatment Patterns and Outcomes for Patients with Adrenocortical Carcinoma Associated with Hospital Case Volume in the United States
}

\author{
Lauren Gratian, $\mathrm{MD}^{1}$, John Pura, $\mathrm{MPH}^{2}$, Michaela Dinan, $\mathrm{PhD}^{3}$, Shelby Reed, $\mathrm{PhD}^{3}$, Randall \\ Scheri, MD ${ }^{4}$, Sanziana Roman, MD ${ }^{4}$, and Julie Ann Sosa, MD ${ }^{3,4}$ \\ Sanziana Roman: sanziana.roman@duke.edu \\ ${ }^{1}$ Division of Endocrinology, Department of Medicine, Duke University School of Medicine, \\ Durham, NC \\ 2Department of Biostatistics, Duke University School of Medicine, Durham, NC \\ ${ }^{3}$ Duke Clinical Research Institute, Durham, NC \\ ${ }^{4}$ Section of Endocrine Surgery, Department of Surgery, Duke University School of Medicine, \\ Durham, NC
}

\begin{abstract}
Background-Adrenocortical carcinoma (ACC) is a rare, aggressive disease with no apparent change in treatment or survival in the United States over the past two decades. Our objective was to determine whether treatment patterns or clinical outcomes vary by hospital case volume.

Methods-Patients with ACC were identified from the National Cancer Database (1998-2011). High-volume centers (HVCs) were defined by a case load of $\geq 4$ cases of primary adrenal malignancy annually, which corresponded to the 90 th percentile. All other facilities were considered low-volume centers (LVCs).
\end{abstract}

Results-A total of 2,765 ACC patients were treated across 1,046 facilities. Compared to patients treated at LVCs, patients treated at HVCs were younger (50 vs. 54 years), with larger tumors (11.2 vs. $10.5 \mathrm{~cm}$ ), and underwent higher rates of surgery (78.8 vs. $73.4 \%)$, radical resection (17.3 vs. $13.9 \%$ ), regional lymph node evaluation (23.2 vs. $18.8 \%$ ), and chemotherapy including mitotane ( 43.8 vs. $31.0 \%$, all $p<0.05$ ). There were no significant differences in median length of stay (5 vs. 5 days), 30-day readmission rates ( $4.0 \%$ for HVCs vs. $3.9 \%$ for LVCs), or 30-day postoperative mortality rates ( $1.9 \%$ for HVCs vs. $3.7 \%$ for LVCs). Median overall survival was 2.0 years for HVCs and 1.9 years for LVCs, $p=0.53$. After adjusting for patient and tumor characteristics, overall survival did not differ significantly between patients treated at HVCs versus LVCs $[\mathrm{HR}=0.89$ (95\% confidence interval 0.70,1.12)].

Conclusions-Treatment at HVCs was associated with more aggressive surgical resection and chemotherapy use. Prognosis remained poor despite more aggressive treatment.

Correspondence to: Sanziana Roman, sanziana. roman@duke.edu.

A portion of these results were presented at the American Association of Endocrine Surgeons 35th Annual Meeting, Boston, MA, April 27, 2014. 
Adrenocortical carcinoma (ACC) is a rare and lethal disease. The estimated annual incidence is $0.5-2.0$ per million. Prognosis is poor, with estimated 5-year survival rates of $82 \%$ for stage I, $61 \%$ for stage II, $50 \%$ for stage III, and $13 \%$ for stage IV. ${ }^{1}$ Complete surgical resection offers the only chance for cure. ${ }^{2-5}$ Although many patients present with resectable disease, up to 75 to $85 \%$ of patients have a relapse after resection. ${ }^{2,6}$ The frequency of recurrence combined with the dismal prognosis associated with advanced disease has led to the development of several adjuvant therapies. ${ }^{7-10}$

Unfortunately, there has been no change in treatment utilization or survival on a population level over the past two decades. ${ }^{11}$ This may be influenced by the fact that about one half of ACC cases in the United States are treated at community hospitals, rather than academic or specialized cancer centers. ${ }^{11}$ Several single institution studies suggest that progress has been made at select quaternary referral centers. ${ }^{12-14}$

Given the rarity of this disease, it is likely that many patients are treated by providers who are not familiar with optimal management strategies. Referral of these patients to specialized centers of care may lead to improvement in oncologic outcomes in these patients. In this study, we described the distribution of facility case volume in the United States and evaluated whether patients with ACC who are treated at high-volume centers (HVCs) have more advanced disease, are treated more aggressively, or have improved short- or long-term clinical outcomes.

\section{METHODS}

\section{Data Source and Patient Selection}

The National Cancer Data Base (NCDB) is a joint project of the American Cancer Society and the Commission on Cancer of the College of Surgeons. The NCDB, established in 1989, is a nationwide, facility-based, comprehensive clinical surveillance resource oncology data set. On the basis of national incidence estimates from the American Cancer Society, the NCDB currently captures $70 \%$ of all newly diagnosed malignancies in the United States annually. ${ }^{15}$

Using the NCDB (1998-2011), patients with ACC were identified on the basis of International Classification of Diseases for Oncology, 2nd and 3rd editions (ICD-O-2/3) for site C74.0-C74.9 and histology 8370.3 (malignant adrenocortical carcinoma). Patients were included only if they had no other primary malignancies. Treating facilities were categorized into HVCs or low-volume centers (LVCs) on the basis of the number of primary adrenal malignancies treated per year. The most common primary adrenal malignancies treated were ACC (60.0\%), malignant pheochromocytoma (15.6\%), and nonspecific carcinoma (11.6 $\%)$. HVCs were defined as centers with an annual case load of $\geq 4$ cases, which corresponded to the 90th percentile of centers performing cases for a given year. This means that centers could fluctuate from being HVCs and LVCs between consecutive years. Radical surgery was defined as partial or total removal of the primary site with removal in continuity of other organs. Debulking was surgery stated to be "debulking." Lymph nodes were considered examined if they were surgically removed or aspirated, and considered positive if malignant cells were identified. Distant metastases were based on the American Joint 
Committee on Cancer clinical staging system. Radiotherapy and chemotherapy were considered administered if they were documented in the first course of treatment. Surgical margins were considered positive if there was evidence of microscopic or macroscopic residual tumor. Overall survival (OS) was defined from time of surgery to time of death or last follow-up. Only patients diagnosed between 1998 and 2006 were included in the OS analysis to allow for at least 5 years of follow-up. This study was determined by the institutional review board to be exempt from oversight as a result of the deidentified nature of the data.

\section{Statistical Analysis}

Baseline characteristics were reported for the study cohort using medians with interquartile ranges (IQR) for continuous variables and frequencies with proportions for categorical variables. Proportions of categorical variables were compared across HVCs and LVCs using Chi square tests, including Fisher's exact tests when the expected cell count was $<1$. Medians of continuous variables were compared across HVCs and LVCs using Wilcoxon rank sum tests. To examine the trend of cases seen at HVCs from 1998 to 2011, a univariable logistic regression model fit the odds of a patient being seen at a $\mathrm{HVC}$ versus $\mathrm{LVC}$ as a function of year of diagnosis.

Short term outcomes, including proportions of patients with 30-day postoperative readmission and 30-day postoperative mortality were compared between center types using univariable logistic regression models with generalized estimating equations (GEE) to account for clustering within single institutions. Mean postoperative length of stay was compared across center type using a univariable linear regression model, also with GEE to account for institutional clustering. OS was first examined in an unadjusted fashion using Kaplan-Meier survival estimates by center type. Survival estimates at 5, 10, and 14 years were compared between HVC and LVC using Wald tests.

A multivariable Cox proportional hazards regression model was then constructed to estimate the adjusted effects of $\mathrm{HVC}$ and other covariates on the risk of death. Before creating the multivariable model, baseline covariates were multiply imputed using chained equations. ${ }^{16}$ The proportion of patients with any missing covariates was approximately $40 \%$. Interaction terms between center type and age, tumor size, race, gender, and nodal status were included in the imputation model, along with linear splines for age and tumor size. A censoring variable for death and the Nelson Aalen estimate of baseline cumulative hazards was also included to preserve the proportional hazards assumption for covariates. ${ }^{17}$ The Cox model estimated the marginal effects of center type, age, race, gender, tumor size, chemotherapy use, radiation use, nodal status, median quarterly income, insurance type, and margin status while accounting for clustering within institutions. Age and tumor size were treated as linear splines with a knot at 35 years and $9 \mathrm{~cm}$, respectively.

\section{RESULTS}

A total of 2765 cases of ACC met study criteria. Fifteen percent (411) of cases were treated at HVCs from 1998 to 2011, and 85 percent (2354) of cases were treated at LVCs. The proportion of cases treated at HVCs increased from $11.6 \%$ in 1998 to $24.6 \%$ in 2011 ( $p<$ 
0.001). There were a total of 1046 centers that treated ACC during our study period. Forty three centers were characterized as HVCs during $\geq 1$ year of the study period, and 1,003 centers were LVCs. Among HVCs, $86 \%$ were academic/research centers, and $14 \%$ were comprehensive community cancer centers. No community cancer centers were identified as HVCs. Patients were found to be similar between the groups with regard to gender, race, and income (Table 1). Patients treated at HVCs were younger than patients treated at LVCs with a median age of 50 years versus 54 years, respectively, less likely to have comorbidities (16.0 vs. $25.6 \%$ ), and had larger median tumor size (11.2 vs. $10.5 \mathrm{~cm}$, all $p<0.001)$. There were $77 \%$ missing data for tumor grade and $84 \%$ missing for clinical stage, which limited our comparison of these variables.

Compared to treatment at LVCs, treatment at HVCs was associated with higher rates of any surgical resection $(78.8$ vs. $73.4 \%, p<0.001)$, radical resection $(17.3$ vs. $13.9 \%, p<$ $0.001)$, open surgical approach (79.5 vs. $66.2 \%, p=0.01)$, regional lymph node evaluation (23.2 vs. $18.8 \%, p=0.05)$, and chemotherapy, including mitotane ( 43.8 vs. $31.0 \%, p<$ $0.001)$.

Among patients who underwent surgery, there were no significant differences in percentage of positive surgical margins (23.2\% for HVCs vs. $25.8 \%$ for LVCs, $p=0.17)$, median length of stay ( 5 vs. 5 days, $p=0.13$ ), 30-day readmission rates (4.0\% for HVCs vs. $3.9 \%$ for LVCs, $p=0.81$ ), or 30-day postoperative mortality rates (1.9\% for HVCs vs. $3.7 \%$ for $\mathrm{LVCs}, p=0.28)$.

Median OS was 2.0 years for HVCs and 1.9 years for LVCs, $p=0.53$ (Fig. 1; Table 2). After adjusting for patient and tumor characteristics, we observed a reduced risk of death in patients treated at HVCs. However, this did not reach statistical significance [HR = 0.89 (95 $\%$ confidence interval $0.70,1.12)$ ]. Cox proportional hazard modeling demonstrated that older age, increasing tumor size up to $9 \mathrm{~cm}$, and positive lymph nodes were associated with reduced survival (Fig. 2). As a sensitivity analysis (not shown), annual case volume was included in the model as a continuous variable and was not significantly associated with risk of death. Interaction between center type and age, tumor size, race, gender, and nodal status were also not statistically significant.

\section{DISCUSSION}

Completeness of surgical resection is the greatest prognostic indicator for patients with ACC. ${ }^{2-5}$ Previous studies have demonstrated that increased surgical volume is an independent predictor of lower rates of postoperative complications and shorter length of stay for patients undergoing adrenalectomy in the United States and Spain. ${ }^{18,19}$ Furthermore, greater surgical experience has been associated with improved oncologic outcomes for patients with ACC. For example, Kerkhofs et al. evaluated patients with stage I to III ACC followed in the National Cancer Registry in the Netherlands. Five-year OS was significantly longer for patients undergoing surgery in a Dutch Adrenal Network (DAN) hospital than for those having surgery in a non-DAN hospital (63 vs. $42 \%$ ). Survival remained significantly different after correction for multiple covariates. ${ }^{20}$ 
Several single institution studies in the U.S. have demonstrated similar results. Kendrick et al. performed a retrospective analysis of patients with ACC treated at the Mayo Clinic from 1980 to 1996 . They found that patients who were treated in 1996 were more likely to undergo curative resections and have improved 5-year OS than institutional historical comparisons. ${ }^{12}$ Grubbs et al. compared patients who underwent primary resection at MD Anderson Cancer Center (MDACC) by three experienced endocrine surgeons and those who underwent resection before referral to MDACC from an outside institution. Patients who underwent surgery at MDACC had higher rates of radical resection and grossly complete tumor resection, and lower rates of histologically involved margins. These patients also had reduced risk of recurrence and superior disease-free survival and OS compared to patients who underwent initial surgical resection at an outside institution. ${ }^{13}$

Our study was the first to evaluate surgical treatment patterns across hospital case volume on a population level within the United States. We also observed that patients treated at HVCs undergo higher rates of any surgical resection, radical resection, and regional lymph node resection compared to patients treated at LVCs.

Up to $85 \%$ of patients with ACC will experience a relapse after radical resection. ${ }^{2,6}$ Therefore, effective adjuvant therapy is of utmost importance in managing this disease. A retrospective multinational study demonstrated improved recurrence-free survival in patients who were routinely administered adjuvant mitotane therapy compared to those who were not. $^{7}$ At the Second Annual International Adrenal Cancer Symposium, an international panel of physicians who specialize in the treatment of ACC recommended use of adjuvant mitotane therapy for patients with increased risk of recurrence after resection. ${ }^{9}$ Patients with advanced disease should be treated with mitotane monotherapy or in combination with various combinations of cisplatin, carboplatin, etoposide, doxorubicin, and streptozosin. ${ }^{8}$ Adjuvant radiotherapy to the tumor bed can also be considered for patients with high-grade adrenal carcinoma, particularly if there is concern for tumor spillage or close margins after surgery. ${ }^{10}$ Despite these recommendations, a population level analysis demonstrated that the type of treatment being provided for patients with ACC in the United States remained unchanged from 1985 through 2005, with the majority of patients undergoing surgery alone. This study also demonstrated no improvement in survival for patients with ACC during this time period. ${ }^{11}$

Our study found that treatment at HVCs was associated with increased use of chemotherapy, including mitotane. This finding is consistent with previous studies that demonstrated increased adjuvant therapy use in tertiary referral centers. For example, Lombardi et al. conducted a survey of patients enrolled in the Italian Registry for ACC. The rate of patients who underwent adjuvant therapy was significantly higher in HVCs compared to LVCs (45.2 vs. $16 \% ; p<0.001)$. In addition, treatment at HVCs was associated with a more aggressive surgical approach, higher rate of lymph node dissection and multiorgan resection. ${ }^{21}$

Fassnacht et al. performed a large multicenter cohort analysis of data maintained by the German ACC registry involving stage II ACC patients who received specialized care within months of initial surgery compared to patients who established specialized care $>4$ months after initial surgery. More patients who registered shortly after diagnosis received adjuvant 
mitotane (53 vs. $16 \%, p<0.001$ ) and had a significantly better 5-year OS compared with patients who enrolled later in their disease course ( 96 vs. $55 \%, p=0.02$ ). However, $67 \%$ of the patients who enrolled later registered only after disease recurrence. In the remaining patients, the recurrence rate was low (21\%), and the 5-year OS was greater than $95 \%$. Therefore, it is unclear whether the apparent survival benefit of early referral was due to more aggressive adjuvant therapy or was the result of a major referral bias among patients referred later in their disease course. ${ }^{22}$

In our study, we did not find an association between hospital case volume and OS despite more aggressive surgical and adjuvant therapy at HVCs. There are several possibilities that could explain the discrepancy between our study and previous findings outlined above. First, we chose to define HVCs as those among the 90th percentile of centers treating primary adrenal malignancies for a given year. We considered whether a more rigorous cut off would have demonstrated more drastic differences in outcomes between center types. However, an additional analysis which examined whether survival was associated with hospital case volume as a continuous variable also was not significant. Second, experience with benign adrenalectomy is not captured in NCDB. Therefore, centers with high benign adrenalectomy volume may be included in the LVC group. Third, prior studies have demonstrated that treatment at HVCs was associated with increased disease-free survival but not OS. ${ }^{21,23}$ The NCDB does not collect information on disease-free survival; therefore, we were unable to evaluate this outcome. Fourth, as a result of a high level of missing data for stage and grade, we were unable to compare these prognostic indicators. It is possible that patients treated at HVCs had more aggressive disease compared to patients treated at LVCs. Finally, there is no way to ensure that patients received all ACC directed care at the reporting facility. Some patients may have undergone initial surgical resection at an outside hospital before referral to the reporting facility. Patients with more aggressive disease may be more likely to be referred to HVCs after disease recurrence, as demonstrated previously by Fassnacht et al. ${ }^{22}$ This potential referral bias may dilute the effect of hospital case volume on patient outcomes in our study.

Our study was subject to limitations inherent to the NCDB database including potential coding errors and the absence of data on mitotic rates, $\mathrm{Ki}-67$ index, disease-specific survival, and disease recurrence. To our knowledge, this is the first population-based study of patients with ACC that encompasses the entire United States and examines the impact of hospital volume on survival in these patients.

In conclusion, our study demonstrates that HVCs appear to treat ACC with more aggressive surgical resection and adjuvant therapy compared to LVCs. However, prognosis remained poor with no significant difference in OS between HVCs and LVCs.

\section{ACKNOWLEDGMENT}

The data used in the study were derived from a deidentified NCDB file. The American College of Surgeons and the Commission on Cancer have not verified and are not responsible for the analytic or statistical methodology employed, or the conclusions drawn from these data by the investigator. 


\section{REFERENCES}

1. Fassnacht M, Johanssen S, Quinkler M, et al. Limited prognostic value of the 2004 International Union Against Cancer staging classification for adrenocortical carcinoma: proposal for a Revised TNM Classification. Cancer. 2009; 115:243-250. [PubMed: 19025987]

2. Pommier RF, Brennan MF. An eleven-year experience with adrenocortical carcinoma. Surgery. 1992; 112:963-970. [PubMed: 1455321]

3. Schulick RD, Brennan MF. Long-term survival after complete resection and repeat resection in patients with adrenocortical carcinoma. Ann Surg Oncol. 1999; 6:719-726. [PubMed: 10622498]

4. Bellantone R, Ferrante A, Boscherini M, et al. Role of reoperation in recurrence of adrenal cortical carcinoma: results from 188 cases collected in the Italian National Registry for Adrenal Cortical Carcinoma. Surgery. 1997; 122:1212-1218. [PubMed: 9426440]

5. Icard P, Goudet $\mathrm{P}$, Charpenay $\mathrm{C}$, et al. Adrenocortical carcinomas: surgical trends and results of a 253-patient series from the French Association of Endocrine Surgeons study group. World J Surg. 2001; 25:891-897. [PubMed: 11572030]

6. Stojadinovic A, Ghossein RA, Hoos A, et al. Adrenocortical carcinoma: clinical, morphologic, and molecular characterization. J Clin Oncol. 2002; 20:941-950. [PubMed: 11844815]

7. Terzolo M, Angeli A, Fassnacht M, et al. Adjuvant mitotane treatment for adrenocortical carcinoma. N Engl J Med. 2007; 356:2372-2380. [PubMed: 17554118]

8. Fassnacht M, Terzolo M, Allolio B, et al. Combination chemotherapy in advanced adrenocortical carcinoma. N Engl J Med. 2012; 366:2189-2197. [PubMed: 22551107]

9. Berruti A, Fassnacht M, Baudin E, et al. Adjuvant therapy in patients with adrenocortical carcinoma: a position of an international panel. J Clin Oncol. 2010; 28:e401-e402. [PubMed: 20567001]

10. Neuroendocrine tumors. NCCN clinical practice guidelines in oncology, Version 1. 2012 http:// www.nccn.org/professionals/physician_gls/f_guidelines.asp\#site.

11. Bilimoria KY, Shen WT, Elaraj D, et al. Adrenocortical carcinoma in the United States: treatment utilization and prognostic factors. Cancer. 2008; 113:3130-3136. [PubMed: 18973179]

12. Kendrick ML, Lloyd R, Erickson L, et al. Adrenocortical carcinoma: surgical progress or status quo? Arch Surg. 2001; 136:543-549. [PubMed: 11343545]

13. Grubbs EG, Callender GG, Xing Y, et al. Recurrence of adrenal cortical carcinoma following resection: surgery alone can achieve results equal to surgery plus mitotane. Ann Surg Oncol. 2010; 17:263-270. [PubMed: 19851811]

14. Vassiolopoulou-Sellin R, Schultz PN. Adrenocortical carcinoma: clinical outcome at the end of the 20th century. Cancer. 2001; 92:1113-1121. [PubMed: 11571723]

15. Winchester DP, Stewart AK, Bura C, Jones RS. The National Cancer Data Base: a clinical surveillance and quality improvement tool. J Surg Oncol. 2004; 85:1-3. [PubMed: 14696080]

16. Raghunathan TW, Lepkowksi JM, Van Hoewyk J, Solenbeger P. A multivariate technique for multiply imputing missing values using a sequence of regression models. Surv Method. 2001; 27:85-95.

17. White IR, Royston P. Imputing missing covariate values for the Cox model. Stat Med. 2009; 28:1982-1998. [PubMed: 19452569]

18. Park HS, Roman SA, Sosa JA. Outcomes from 3144 adrenalectomies in the United States. Which matters more, surgeon volume or specialty? Arch Surg. 2009; 144:1060-1067. [PubMed: 19917944]

19. Villar JM, Moreno P, Ortega J, et al. Results of adrenal surgery. Data of a Spanish national survey. Langenbecks Arch Surg. 2010; 395:837-843. [PubMed: 20658299]

20. Kerkhofs TM, Verhoeven RH, Bonjer HJ, et al. Surgery for adrenocortical carcinoma in the Netherlands: analysis of the national cancer registry data. Eur J Endocrinol. 2013; 169:83-89. [PubMed: 23641018]

21. Lombardi CP, Raffaelli M, Boniardi M, et al. Adrenocortical carcinoma: effect of hospital volume on patient outcome. Langenbecks Arch Surg. 2012; 397:201. [PubMed: 22069043] 
22. Fassnacht M, Johanssen S, Fenske W, et al. Improved survival in patients with stage II adrenocortical carcinoma followed up prospectively by specialized centers. J Clin Endocrinol Metab. 2010; 95:4925-4932. [PubMed: 20668036]

23. Else T, Williams AR, Sabolch A, Jolly S, Miller BS, Hammer GD. Adjuvant therapies and patient and tumor characteristics associated with survival of adult patients with adrenocortical carcinoma. J Clin Endocrinol Metab. 2014; 99:455-461. [PubMed: 24302750] 


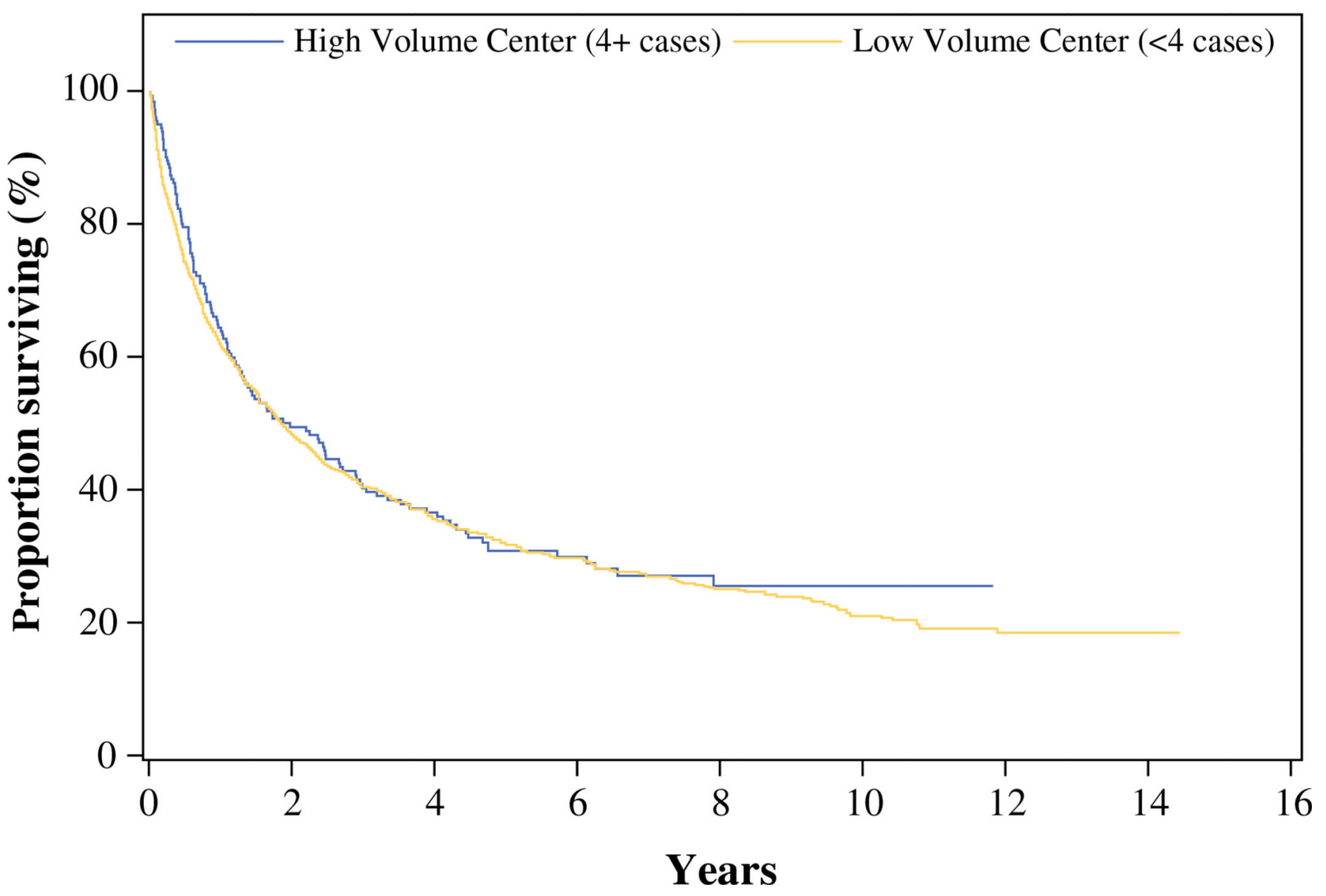

FIG. 1.

Unadjusted overall survival by hospital case volume for patients undergoing treatment for ACC 


\begin{tabular}{|c|c|c|c|}
\hline High volume center & $\longmapsto$ & 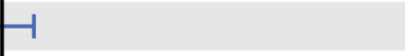 & $0.89(0.71-1.10)$ \\
\hline Age at most $35 \mathrm{yrs}$ & $\longmapsto$ & -1 & $0.96(0.82-1.12)$ \\
\hline Age $>35$ yrs & & 바ㅁㅓㅓ & $1.10(1.06-1.14)$ \\
\hline Non-Caucasian race & $\longmapsto$ & H & $0.83(0.63-1.09)$ \\
\hline Male & $\longmapsto$ & $H$ & $0.93(0.79-1.10)$ \\
\hline Size at most $9 \mathrm{~cm}$ & & $\mathrm{H}$ 범 & $1.11(1.05-1.17)$ \\
\hline Size $>9 \mathrm{~cm}$ & & 1 & $1.01(0.99-1.03)$ \\
\hline \multirow[t]{3}{*}{ Nodal status present } & & $\longmapsto-1$ & $1.25(1.05-1.49)$ \\
\hline & Lower risk of death & \multicolumn{2}{|l|}{ Higher risk of death } \\
\hline & 0.5 & $\begin{array}{llll}1 & & 1 & 1 \\
1.5 & 2 & 2.5 & 3\end{array}$ & \\
\hline
\end{tabular}

FIG. 2.

Factors associated with overall survival for patients treated for ACC. Age > 35 years, positive lymph node status, and increasing tumor size up to $9 \mathrm{~cm}$ were associated with increased risk of death 


\section{TABLE 1}

Demographic, disease, and treatment characteristics of patients undergoing treatment for $\mathrm{ACC}^{a}$

\begin{tabular}{|c|c|c|c|}
\hline Characteristic & $\operatorname{HVC}(n=411)$ & $\operatorname{LVC}(n=2354)$ & $p$ \\
\hline Age, year, median (Q1, Q3) & $50(41,60)$ & $54(43,65)$ & $<0.0001$ \\
\hline Female gender $(\%)$ & 57.4 & 59.9 & 0.34 \\
\hline Race $(\%)$ & & & 0.57 \\
\hline White & 87.4 & 87.3 & \\
\hline Black & 8.2 & 9.2 & \\
\hline Other & 4.4 & 3.5 & \\
\hline Charlson/Deyo score $\geq 1(\%)$ & 16.0 & 25.6 & 0.0002 \\
\hline Income $(\%)$ & & & 0.26 \\
\hline$<30,000$ & 10.2 & 12.8 & \\
\hline $30,000-45,900$ & 44.7 & 46.4 & \\
\hline $46,000+$ & 45.1 & 40.8 & \\
\hline Primary payer (\%) & & & $<0.0001$ \\
\hline Not insured & 2.7 & 5.1 & \\
\hline Private insurance & 57.4 & 58.2 & \\
\hline Medicare & 0.5 & 0.8 & \\
\hline Medicaid & 18.5 & 26.4 & \\
\hline Other & 20.9 & 9.5 & \\
\hline Tumor size, cm, median (Q1, Q3) & $11.2(8.0,16.0)$ & $10.5(7.1,15.0)$ & 0.0040 \\
\hline Distant metastasis present (\%) & 25.5 & 35.3 & $<0.0001$ \\
\hline Grade (\%) & & & 0.02 \\
\hline 1 & 11.1 & 16.1 & \\
\hline 2 & 25.3 & 16.1 & \\
\hline 3 & 38.4 & 49.4 & \\
\hline 4 & 25.3 & 18.5 & \\
\hline NCDB analytic stage (\%) & & & 0.0003 \\
\hline 1 & 4.3 & 6.3 & \\
\hline 2 & 39.8 & 30.8 & \\
\hline 3 & 15.1 & 17.3 & \\
\hline 4 & 39.8 & 45.5 & \\
\hline Hospital type (\%) & & & $<0.0001$ \\
\hline Community cancer program & 0.0 & 8.7 & \\
\hline Comprehensive community cancer program & 5.7 & 48.8 & \\
\hline Academic/research program & 94.3 & 42.5 & \\
\hline Surgery type (\%) & & & $<0.0001$ \\
\hline No surgery & 21.2 & 26.6 & \\
\hline Local tumor surgery & 1.9 & 4.9 & \\
\hline Simple/partial resection & 9.5 & 11.2 & \\
\hline Total resection & 39.2 & 40.0 & \\
\hline Debulking & 1.0 & 1.4 & \\
\hline
\end{tabular}




\begin{tabular}{lrrr}
\hline Characteristic & HVC $(\boldsymbol{n}=\mathbf{4 1 1})$ & $\mathbf{L V C}(\boldsymbol{n}=\mathbf{2 3 5 4})$ & $\boldsymbol{p}$ \\
\hline Radical surgery & 17.3 & 13.9 & \\
Surgery NOS & 10.0 & 2.0 & \\
Surgical approach (\%) & & 0.01 \\
Robotic assisted & 1.3 & 5.8 & \\
Endoscopic/laparoscopic & 16.7 & 23.6 & \\
Endoscopic convert to open & 2.6 & 4.4 & \\
Open & 79.5 & 66.2 & \\
Regional LNs examined (\%) & 23.2 & 18.8 & 0.05 \\
Positive regional LNs (\%) & 6.6 & 5.3 & 0.33 \\
Positive surgical margins (\%) & 23.2 & 25.8 & 0.17 \\
Chemotherapy administered (\%) & 43.8 & 31.0 & $<0.0001$ \\
Radiotherapy administered (\%) & 12.9 & 10.6 & 0.17 \\
\hline
\end{tabular}

$A C C$ adrenocortical carcinoma, $H V C$ high-volume center, $L V C$ low-volume center, $N C D B$ National Cancer Data Base, $N O S$ not otherwise specified, $L N$ lymph node

${ }^{a}$ Missing data for each variable are $<5 \%$ except for the following: Charlson/Deyo score, $30 \%$; income, $6 \%$; distant metastasis, $84 \%$; grade, $77 \%$; stage, $84 \%$; surgical approach, $83 \%$; LNs examined, $9 \%$; LN positive, $9 \%$; surgical margins, $13 \%$ 
TABLE 2

Kaplan-Meier estimates of unadjusted OS by hospital case volume for patients undergoing treatment for ACC

\begin{tabular}{llll}
\hline $\begin{array}{l}\text { End point OS } \\
\mathbf{9 5} \% \text { CI })\end{array}$ & $\begin{array}{l}\text { Low-volume } \\
\text { center }\end{array}$ & $\begin{array}{l}\text { High-volume } \\
\text { center }\end{array}$ & $\boldsymbol{p}$ value \\
\hline 5 years & $31.8 \%(29.2,34.3)$ & $30.7 \%(24.0,37.7)$ & 0.782 \\
10 years & $20.8 \%(18.1,23.6)$ & $25.5 \%(18.6,32.9)$ & 0.227 \\
Median survival, years & $1.9(1.7,2.1)$ & $2.0(1.3,2.9)$ & 0.534 \\
\hline
\end{tabular}

$O S$ overall survival, $C I$ confidence interval, $A C C$ adrenocortical carcinoma 